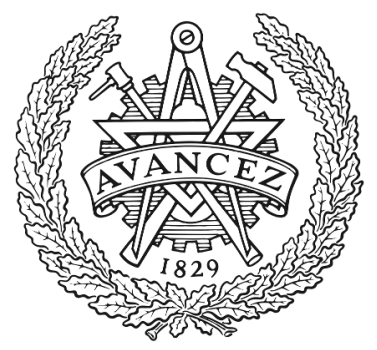

CHALMERS

UNIVERSITY OF TECHNOLOGY

\title{
Optical Rotation and Thermometry of Laser Tweezed Silicon Nanorods
}

Downloaded from: https://research.chalmers.se, 2023-04-26 12:27 UTC

Citation for the original published paper (version of record):

Karpinski, P., Jones, S., Jungová, H. et al (2020). Optical Rotation and Thermometry of Laser

Tweezed Silicon Nanorods. Nano Letters, 20(9): 6494-6501.

http://dx.doi.org/10.1021/acs.nanolett.0c02240

N.B. When citing this work, cite the original published paper. 


\title{
Optical Rotation and Thermometry of Laser Tweezed Silicon Nanorods
}

\author{
Pawel Karpinski,* Steven Jones, Hana Sípová-Jungová, Ruggero Verre, and Mikael Käll* \\ Cite This: Nano Lett. 2020, 20, 6494-6501 \\ Read Online
}

ABSTRACT: Optical rotation of laser tweezed nanoparticles offers a convenient means for optical to mechanical force transduction and sensing at the nanoscale. Plasmonic nanoparticles are the benchmark system for such studies, but their rapid rotation comes at the price of high photoinduced heating due to Ohmic losses. We show that Mie resonant silicon nanorods with characteristic dimensions of $\sim 220 \times 120 \mathrm{~nm}^{2}$ can be optically trapped and rotated at frequencies up to $2 \mathrm{kHz}$ in water using circularly polarized laser light. The temperature excess due to heating from the trapping laser was estimated by phonon Raman scattering and particle rotation analysis. We find that the silicon nanorods exhibit slightly improved thermal characteristics compared to $\mathrm{Au}$

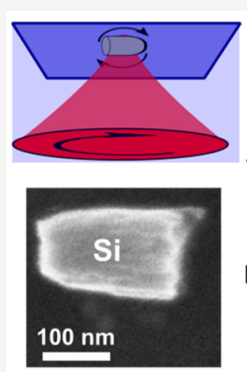
Silicon nanorods nanorods with similar rotation performance and optical resonance anisotropy. Altogether, the results indicate that silicon nanoparticles have the potential to become the system of choice for a wide range of optomechanical applications at the nanoscale.

KEYWORDS: optical tweezers, optical rotation, nanorods, silicon, thermometry, Raman scattering
$\mathrm{O}$ ptical manipulation experiments on nanoparticles have to a large extent focused on noble metal nanostructures that support surface-plasmon resonances, which enhance light-matter interactions and therefore typically amplify optical forces. ${ }^{1,2}$ Additionally, the advanced colloidal synthesis methods available for noble metals, in particular gold, mean that plasmonic nanoparticles can be made with highly customized and extremely well-defined shapes, sizes, and resonance properties. This has driven a plethora of applications, including nanoparticle-based photothermal treatment, ${ }^{3,4}$ molecular sensing, ${ }^{5}$ and drug delivery, ${ }^{6-8}$ but it has also enabled a wide range of optical manipulation studies of plasmonic nanoparticles. ${ }^{1,9,10}$ However, the enhanced plasmonic light-matter interaction comes at the price of extensive laser-induced heating of the nanostructure, ${ }^{11,12}$ which in extreme cases may even lead to vapor formation at the surface of a trapped particle. ${ }^{13-16}$ Photothermal heating is a particular concern when dealing with sensitive biological systems, where it may lead to protein denaturation and cell death, for example. ${ }^{17}$ This has motivated investigations of alternative nanoparticle systems that combine enhanced optical interaction with reduced susceptibility to photothermal heating. Nanoparticles made of high index dielectric (HID) materials, in particular silicon, appear as one of the most interesting candidates in this respect. Because of the large refractive index, silicon nanoparticles can support geometric Mie resonances with quality factors similar to plasmonic nanoparticles at visible/NIR wavelengths but with significantly lower optical absorption, especially in the NIR range. ${ }^{18,19}$
Several optical manipulation studies of $\mathrm{Si}$ nanowires have been reported in the literature (see, e.g., refs 20-22), but so far, only a few works have dealt with true nanoparticles, i.e., particles with all dimensions substantially smaller than 1 $\mu \mathrm{m}^{23-27}$ One reason for this is the limited availability of colloidal suspensions suitable for optical tweezing experiments, although $\mathrm{Si}$ nanospheres with good crystalline quality and narrow size distribution have recently become available. ${ }^{28} \mathrm{~A}$ greater variety of available shapes could significantly expand the range of possible applications, as has been the case with plasmonic nanoparticles. Toward this end, we recently developed a lithography-based synthesis method that can be used to produce sample quantities of Si colloidal solutions with customized particle shapes and narrow size distributions. $^{25,29}$

In this work, we demonstrate and discuss optical rotation of $\mathrm{Si}$ nanorods trapped by optical tweezers, i.e., the classical single-beam gradient-force trap. ${ }^{30}$ Optical rotation offers a powerful means to probe viscoelastic properties of complex fluids in confined geometries ${ }^{31,32}$ and constitute a basis for the development of optical micromachinery and rotary nanomotors ${ }^{10,33,34}$ The rotation effect requires transfer of spin angular momentum from the incident laser beam to the

Received: May 28, 2020

Revised: July 24, 2020

Published: July 27, 2020 
a)

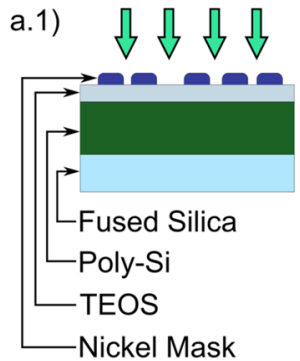

a.2)
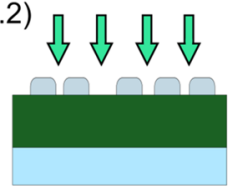

a.3)

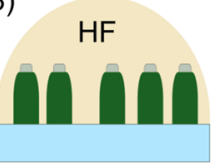

b)

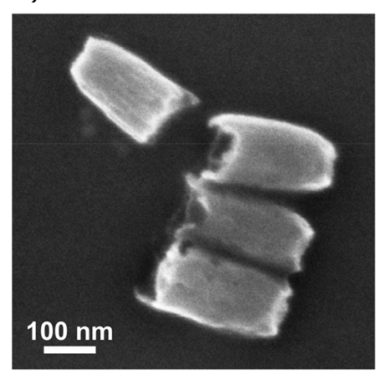

c)

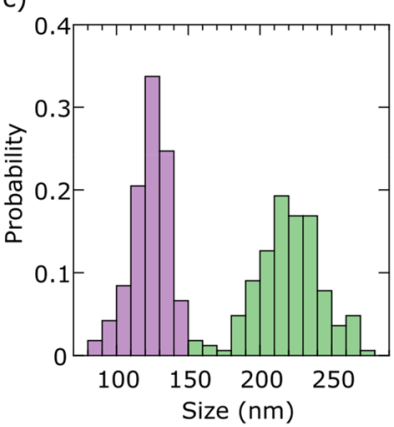

a.4)

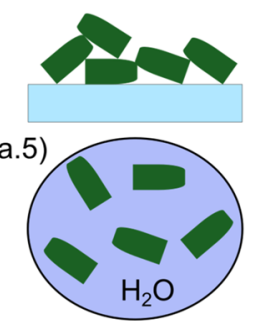

d)

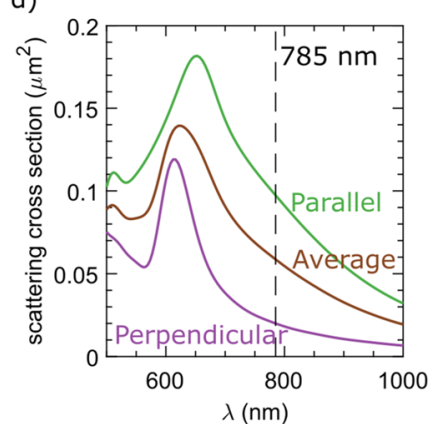

Figure 1. Fabrication and characterization of colloidal Si nanorods. (a) Sketch of the procedure used to fabricate Si particles with a specific shape and size. (b) Scanning electron microscopy images of representative Si nanorods. (c) Histograms of particle diameters (violet) and lengths (green) obtained from analysis of SEM images. (d) Simulated scattering spectra of Si nanoparticles for the cases when the polarization of the incident plane wave is perpendicular or parallel to the long axis of the nanoparticle. The vertical dashed line indicates the laser wavelength used for optical tweezing and rotation.

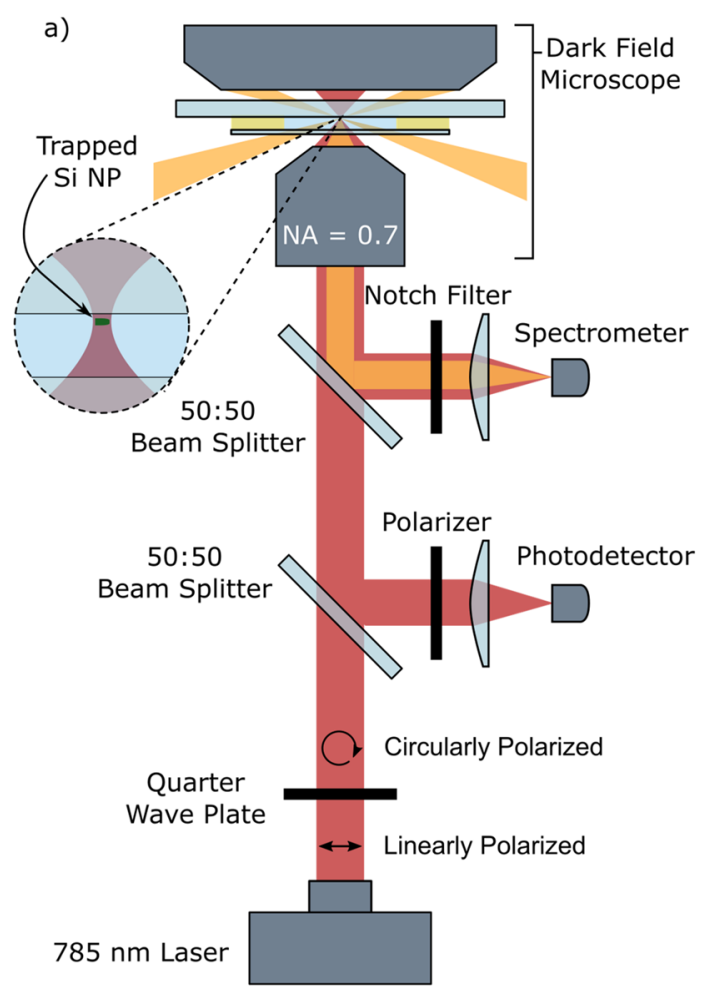

b)

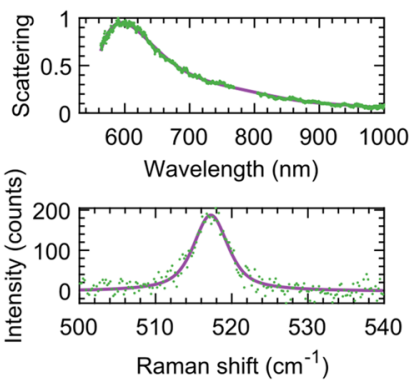

d)

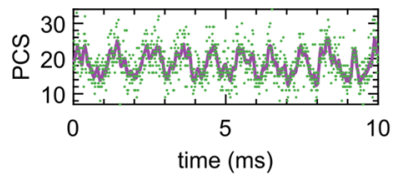

e)

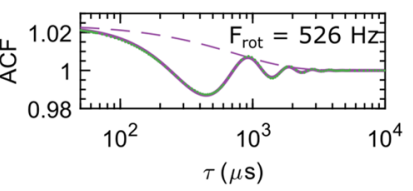

f)

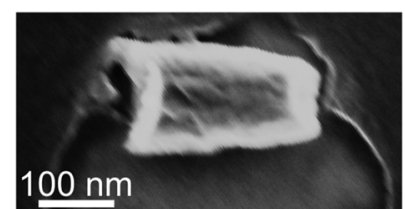

Figure 2. Optical rotation and scattering spectroscopy of laser tweezed resonant Si nanorods. (a) Schematic of the experimental setup, which is based on circularly polarized $785 \mathrm{~nm}$ laser tweezers; white-light dark-field (DF) illumination; linearly polarized photon correlation spectroscopy; and elastic (Rayleigh) or inelastic (Raman) scattering spectroscopy. (b-f) Experimental results obtained from the same optically trapped and rotated Si nanorod: (b) dark-field scattering spectrum; (c) Raman spectrum covering the region around the $\sim 520 \mathrm{~cm}^{-1} \mathrm{Si}$ optical phonon together with Lorentzian fit (purple line); (d) scattering intensity versus time during optical rotation (the purple line is a guide to the eye only) and (e) the corresponding autocorrelation function, showing decaying oscillations characteristic for damped rotational motion; (f) SEM image of the particle, obtained after the nanorod had been printed on the substrate by increasing the laser power. The purple line in part e shows the full analytical fit to the experimental ACF data, while the dashed line shows the exponentially decaying component extracted from the fit. 

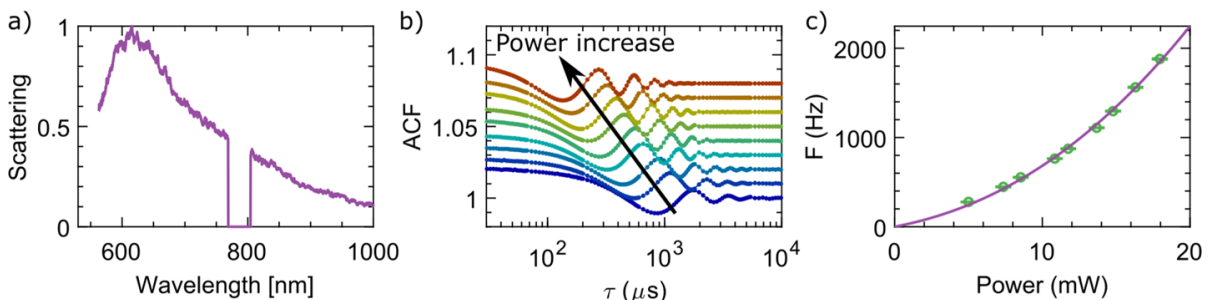

Figure 3. Analysis of rotational dynamics of a trapped Si nanoparticle through photon correlation spectroscopy. (a) Dark-field scattering spectra of the trapped Si nanoparticle. (b) Autocorelation function versus increasing applied laser power (vertically offset for clarity) and (c) corresponding rotation frequency versus laser power as extracted from the measured autocorrelation data (error bars represent $95 \%$ confidence bounds) together with fit to the analytical $f_{\text {rot }}(P)$ function described in the text (purple line).

particle, which in turn requires that the laser trap is circularly polarized and that the trapped particle somehow alters the polarization state of the beam. The most effective way of achieving this is to utilize particles with anisotropic shapes, such as nanorods, which in the optimal case exhibit linearly polarized scattering. ${ }^{33}$ Based on this approach, we show that "shape-birefringent" Si nanorods can be rotated at high frequencies comparable to plasmonic nanoparticles. Moreover, we show that the degree of photothermal heating of the rotating particles can be monitored through both rotation analysis and Raman spectroscopy. A quantitative comparison of the results with previous reported studies of optical rotation of submicron particles is presented at the end of the article.

\section{METHODOLOGY, RESULTS, AND DISCUSSION}

Aqueous colloidal solutions of $\mathrm{Si}$ nanorods were prepared using the two-step fabrication process schematically illustrated in Figure 1a. Dense and uniform layers of in-plane cylindrical nickel nanoparticles are first fabricated on a poly-Si film deposited on a fused silica substrate using a hole-mask colloidal lithography method. ${ }^{25,29}$ The $\mathrm{Ni}$ particles are used as an etching mask that allows the polycrystalline silicon film underneath to be shaped into nanorods by means of directional etching. The Si nanorods can then be detached from the substrate via selective etching of the fused silica substrate using hydrofluoric acid (HF), followed by purification from the etchant, sonication, and dispersion into water (see the Methods for details). The etching procedures lead to slightly tapered shapes and sometimes to somewhat fragmented base surfaces (Figure $1 \mathrm{~b}$ ). The average dimensions of the nanorods can be tuned by varying the thickness of poly$\mathrm{Si}$ film and the diameter of Ni nanoparticles. Based on optical simulations and fabrication considerations, we aimed at an average nanorod length of $L=220 \mathrm{~nm}$ and a diameter of $D=$ $120 \mathrm{~nm}$. Statistics from scanning electron microscopy (SEM) images revealed a resulting average length of $L=219 \pm 23 \mathrm{~nm}$ and diameter of $D=123 \pm 13 \mathrm{~nm}$ (Figure 1c).

Figure 1d shows finite difference time domain (FDTD) electrodynamics simulations of scattering spectra for a $120 \times$ $220 \mathrm{~nm}^{2} \mathrm{Si}$ nanorod in water for the case when its long axis is oriented perpendicular to the light propagation direction. We have assumed a perfectly cylindrical shape for simplicity. Figure $1 \mathrm{~d}$ reveals that the particle shows strong scattering anisotropy because of its elongated shape. At the optical tweezers wavelength used in the experiments, $785 \mathrm{~nm}$, the scattering is $\sim 5 \times$ stronger for incident light polarized parallel to the particle long axis compared to the orthogonal polarization configuration. The shape birefringence associated with the optical anisotropy leads to efficient transfer of spin angular momentum from an incident circularly polarized trapping beam, since the light scattered out from the particle will be essentially linearly polarized. This is in turn a prerequisite for efficient optical rotation of a trapped particle.

Single $\mathrm{Si}$ nanorods were optically trapped using optical tweezers constructed around an inverted optical microscope equipped with various means for spectroscopic characterization (Figure 2a). The laser tweezers are based on a circularly polarized $785 \mathrm{~nm}$ laser beam focused by a low-numericalaperture $(\mathrm{NA}=0.7)$ microscope objective. This ensures nearly perfect circular polarization in the optical trap and allows for facile dark-field (DF) observation, but the low NA reduces the gradient force in the direction of the optical axis such that stable trapping in three dimensions is not possible. The nanorods are therefore trapped in $2 \mathrm{D}$ against the upper coverglass of the thin liquid cell used to confine the diluted colloid suspension. The setup offers multiple means for analyzing the trapped particle and its rotational dynamics. This is illustrated in Figure $2 b-f$, which shows results obtained from one and the same trapped Si nanorod.

The DF arrangement allows for measurements of elastic scattering spectra of a trapped nanoparticle during rotation experiments. The DF spectrum shown in Figure $2 \mathrm{~b}$ exhibits a pronounced Mie resonance peak at $\sim 600 \mathrm{~nm}$ that agrees well with the averaged simulated scattering spectrum shown in Figure 1d. Moreover, with the DF illumination turned off, it is possible to obtain Raman spectra of the trapped particle by using the $785 \mathrm{~nm}$ trapping laser as the Raman excitation source. This is illustrated in Figure 2c, which highlights the region around the characteristic transverse optical Si phonon at $\sim 520 \mathrm{~cm}^{-1}$. Apart from being a highly sensitive spectroscopic fingerprint of the material composition and structure, the $\sim 520$ $\mathrm{cm}^{-1}$ phonon can be used to estimate the internal temperature of the trapped nanorod, as discussed further below. Third, by sampling part of the backscattered laser light with high time resolution, one can perform localized photon correlation spectroscopy on the trapped particle (PCS, Figure 2d). Because of the shape anisotropy of the trapped nanorod, the backscattered signal will be highly polarized (cf. Figure 1d). Thus, by filtering the signal through a linear polarizer placed in front of the detector, any orientational fluctuation of the trapped nanorod will show up as a fluctuation in the PCS signal. By analyzing the PCS autocorrelation function, one can then obtain information on the time-averaged rotational dynamics of the trapped particle (Figure $2 \mathrm{e}$ ). ${ }^{35}$ Finally, when optical measurements have been concluded, the trapped particle can be printed on the upper cover-glass of the sample cell by an increase in laser power and subsequently analyzed with SEM (Figure $2 \mathrm{f}$ ). However, this procedure requires a high salinity $(\sim 10 \mathrm{mM} \mathrm{NaCl})$ of the sample solution such that 

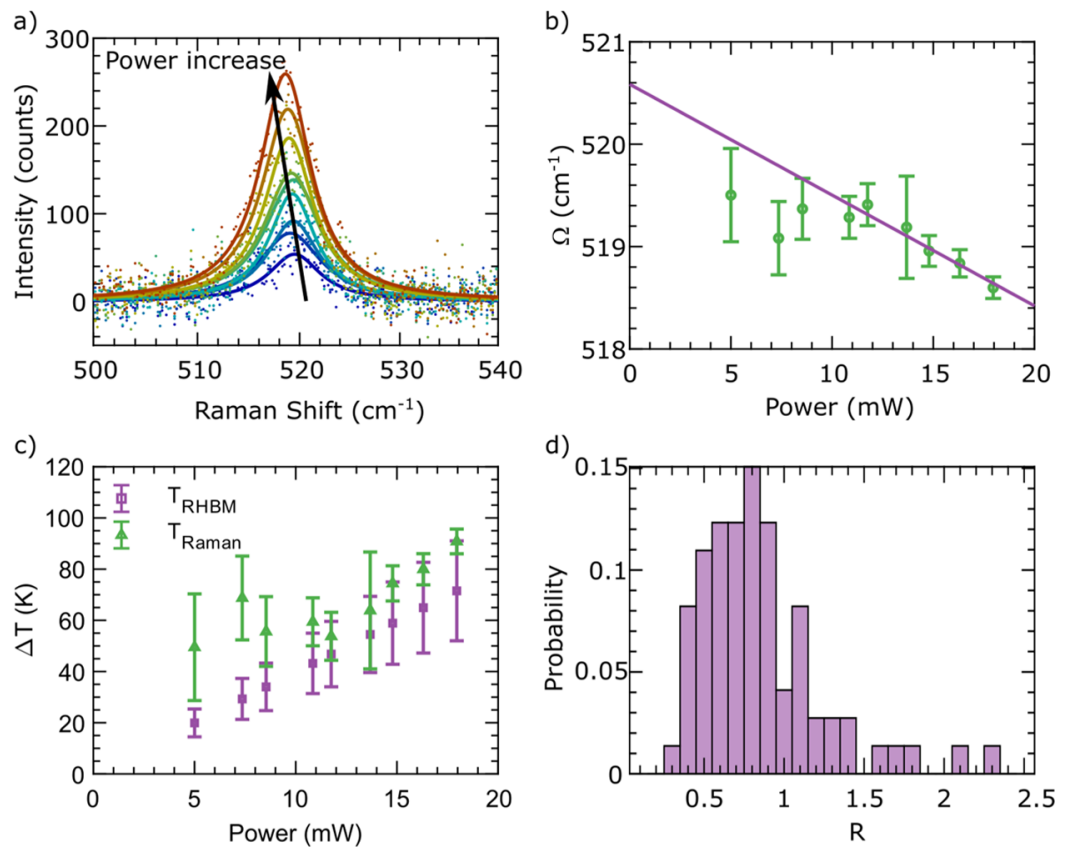

Figure 4. Temperature estimates of an optically trapped and rotated Si nanorod. (a) Stokes-shifted Raman spectra together with Lorentzian fits to the $520 \mathrm{~cm}^{-1}$ optical phonon. All spectra are measured using the $785 \mathrm{~nm}$ laser trap as an excitation source and using the same integration time. The arrow indicates phonon softening due to photothermal heating for increasing laser power. (b) Raman peak position $\Omega$ versus laser power together with linear extrapolation of high-power data $(P>10 \mathrm{~mW})$ to $P=0$. (c) Temperature increment versus laser power extracted from Raman peak shift and rotation frequency analysis. Error bars represent $95 \%$ confidence limits obtained from fits to experimental data. (d) Histogram of the ratios between temperature increments extracted from rotational motion and Raman phonon analysis.

electrostatic repulsion between the trapped particle and the cover glass is effectively screened. This in turn results in salt deposits around the laser printed particle, as can be observed in Figure 2f. Figures S1 and S2 in the Supporting Information show results for a number of trapped nanorods, confirming the overall reproducibility of our results.

We next investigated how the nanorod rotation performance varied with applied laser power. These experiments had to be performed without adding salt to the solution, meaning that the particles could not be laser-printed for subsequent SEM imaging. However, the full laser power range could now to be utilized for tracking how the different measurables (DF, PCS, and Raman) varied with laser power. In the following, we display results obtained for one representative nanorod, but the conclusions are based on combined observations from many different particles (summarized in Figure S4 in the Supporting Information).

Figure 3a shows the measured DF spectrum of the trapped nanorod. The spectrum is in good agreement with the FDTD scattering calculations in Figure 1d, indicating that the particle is representative for the colloid batch (i.e., $L \approx 220 \mathrm{~nm}, D \approx$ $120 \mathrm{~nm}$ ). From the rotational autocorrelation functions (Figure $3 \mathrm{~b}$ ), we can extract the average rotation frequency $f_{\text {rot }}$ (Figure $3 \mathrm{c}$ ) and the corresponding decay constant $\tau_{0}$ due to rotational Brownian motion (Figure S3) by a fit to $\operatorname{ACF}(\tau)=A$ $+B \exp \left(-\tau / \tau_{0}\right) \cos \left(4 \pi f_{\text {rot }} \tau\right){ }^{10,36}$ The results show that $f_{\text {rot }}$ increases with laser power in a supralinear fashion, reaching $\sim 2$ $\mathrm{kHz}$ at the maximum applied power of $P=18 \mathrm{~mW}$ (Figure 3c).

An analysis of the $f_{\text {rot }}(P)$ data in Figure $3 \mathrm{c}$ allows us to estimate the local temperature around the trapped nanorod as a function of laser power. The average rotation frequency of an optically rotated nanorod is determined by the applied optical torque and the Stokes drag, yielding $f_{\text {rot }} \propto P / \eta$, where $P$ is the applied laser power and $\eta$ is the viscosity of the surrounding fluid. We assume the viscosity of bulk water with temperature dependence $\eta(T)=\eta_{0} \exp \left(\frac{A}{T-T_{\mathrm{vF}}}\right)$, with $\eta_{0}=24.2 \mu \mathrm{Pa} \cdot \mathrm{s}, A=$ $570.56 \mathrm{~K}$, and $T_{\mathrm{vF}}=140 \mathrm{~K}^{37}$ The temperature of water close to the heated nanorod will increase in proportion to applied laser power as $T=T_{0}+k P$, where $T_{0}=295 \mathrm{~K}$ is the bath temperature and $k$ is a constant. This yields $f_{\text {rot }}(P) \propto P \exp \left(\frac{-A}{T_{0}-T_{\mathrm{vF}}+k P}\right)$, which can be used to fit experimental data in order to extract $k$ and thereby $T(P)$. In the following, we compare the temperature extracted from the rotational data in Figure $3 \mathrm{c}$ with Raman thermometry data obtained from the same nanorod.

Raman thermometry of $\mathrm{Si}$ nanoparticles is based on the temperature dependent vibrational frequency $\Omega(T)$ of the 520 $\mathrm{cm}^{-1}$ optical phonon, which softens with increasing temperature due to lattice expansion and anharmonicity effects. ${ }^{38,39}$ Within a broad interval between room temperature $T_{0}$ and $\sim 550 \mathrm{~K}$, the phonon frequency varies almost linearly with temperature, $\Omega(T)=\Omega\left(T_{0}\right)+a\left(T-T_{0}\right)$, with $a=-0.0218$ $\mathrm{cm}^{-1} \mathrm{~K}^{-1}$. ${ }^{38}$ However, the phonon frequency also depends sensitively on crystalline quality, with polycrystalline $\mathrm{Si}$ in general showing softer $\Omega\left(T_{0}\right)$ than monocrystalline silicon due to intrinsic residual stress. This means that one needs at least one reference measurement at a known temperature for absolute Raman thermometry (Figure S5). ${ }^{40,41}$

Figure 4a shows Raman spectra covering the $520 \mathrm{~cm}^{-1}$ phonon for the same trapped nanorod, as analyzed in Figure 3. Lorentzian fits to the data yield the phonon frequency $\Omega(P)$ versus laser power $P$ (Figure $4 \mathrm{~b})$. We do not know $\Omega(P=0)$, since we need a certain threshold power to be able to trap the particle. However, the data at high powers $(P>10 \mathrm{~mW})$ reveal a rather clear linear softening due to the photothermal heating 
Table 1. Characteristics of Submicron Particles Optically Rotated by Circularly Polarized Laser Tweezers

$\begin{array}{lllll}\text { material }(\mathrm{ref}) & \mathrm{Si} \text { (this work) } & \mathrm{Au}^{33} & \mathrm{Au}^{33} & \mathrm{Au}^{10} \\ L(W)(\mathrm{nm}) & \sim 220(120) & \sim 134(65) & \sim 173(65) & \sim \\ g & \sim 0.41 & \sim 0.36 & \sim 0.29 & 1 \\ \lambda_{\text {laser }}(\mathrm{nm}) & 785 & 830 & 830 & 830 \\ \frac{\partial T}{\partial P}(\mathrm{~K} / \mathrm{mW}) & \sim 3 & \sim 5 & \sim 25 & \sim 800 \\ \left.\frac{\partial f_{\text {rot }}}{\partial P}\right|_{P=0}[\mathrm{~Hz} / \mathrm{mW}] & \sim 40 & \sim 160 & \sim 1000 & \sim 20 \\ \frac{\partial M_{\text {opt }}}{\partial P}(10-18 \mathrm{~N} \cdot \mathrm{m} / \mathrm{W}) & \sim 3.3 & \sim 2.6 & \sim 30 & \sim 24\end{array}$

that can be extrapolated to $P=0$, when the particle is assumed to be at $T_{0}=295 \mathrm{~K}$. We can then estimate the particle temperature as $T(P)=T_{0}+a^{-1}(\Omega(P)-\Omega(0))$. Figure $4 \mathrm{c}$ summarizes the temperature increments obtained in this way together with the data from the rotational temperature determination obtained simultaneously from the same particle, as described above. It is clear that the two data sets are in good general agreement, both indicating $\Delta T \approx 80 \mathrm{~K}$ at the highest applied laser power.

Figure S4 in the Supporting Information summarizes data for eight different nanorods measured and analyzed by the same methodology as in Figure 3 and Figure 4. The results are in overall good agreement, though some variation between different nanorods is apparent. For example, we find that the maximum rotation frequency varies between $\sim 1$ and $\sim 2 \mathrm{kHz}$ at $\sim 18 \mathrm{~mW}$, while the corresponding maximum temperature increment varies between $\sim 30$ and $\sim 90 \mathrm{~K}$. The variation is likely due to small differences in particle shape and size, which influence both the degree of viscous rotational friction (Stokes drag) as well the particle optical properties. The latter in turn determines the strength of the optical torque as well as the degree of optothermal heating for a given laser power.

As seen in Figure 4c, there is a tendency for the Raman thermometry to produce slightly higher temperatures than the rotational analysis. This is expected, because the Raman signal originates from the hot interior of the particle, where light absorption occurs, while the rotation phenomenon probes the fluid viscosity just outside the particle via rotational hot Brownian motion (RHBM), ${ }^{42}$ that is, the stochastic thermal fluctuations in orientation experienced by a particle that is hotter than its surrounding. We have previously shown that $T_{\text {RHBM }}$ corresponds to the actual temperature 10-15 nm outside the surface of an optically rotated plasmonic nanorod. ${ }^{15,36}$ In Figure $4 \mathrm{~d}$, we show a histogram over the ratios $R=\Delta T_{\text {Raman }} / \Delta T_{\text {RHBM }}$ for all measured particles and laser powers. The variation is substantial due to large error bars in $\Delta T_{\text {Raman }}$ obtained at low $P$, for which the Raman signal is weak. However, there is a rather clear peak at $R \approx 0.75$, which happens to be the theoretically expected ratio between $T_{\text {RHBM }}$ and the surface temperature excess of a spherical particle performing RHBM (though this result is obtained under the simplifying approximation of a temperature independent viscosity).

To put the results presented here in perspective, Table 1 summarizes important rotational and thermal characteristics for the Si nanorods together with corresponding data from previously published investigations of optically rotated submicron particles, including two classes of single crystal $\mathrm{Au}$ nanorods, ${ }^{33}$ with widths of $\sim 65 \mathrm{~nm}$ and lengths of $\sim 134$ and $\sim 175 \mathrm{~nm}$, respectively; $\sim 400 \mathrm{~nm}$ diameter Au colloids; ${ }^{9}$ and $\sim 800 \mathrm{~nm}$ diameter spherical vaterite $\left(\mathrm{CaCO}_{3}\right)$ particles. ${ }^{43}$
These particle types also illustrate different means to extract torque from a circularly polarized laser beam, i.e., through polarization conversion caused by material birefringence (vaterite); through absorption (400 nm Au); and mainly through polarized scattering (shape birefringence, $\mathrm{Si}$ and $\mathrm{Au}$ nanorods).

We first focus on the propensity for photothermal heating, $k$ $=\mathrm{d} T / \mathrm{d} P$, and on the rotation frequency induced per incident power unit, $\mathrm{d} f_{\text {rot }} / \mathrm{d} P$. The latter needs to be extrapolated to $P=$ 0 to avoid the influence from differences in photothermal heating between the different particle types. The Si nanorods obviously fall in an intermediate class between the extreme cases of vaterite, which exhibits negligible heating but also very low $\mathrm{d} f_{\text {rot }} / \mathrm{d} P$, and the high-aspect-ratio $\mathrm{Au}$ nanorod, which shows record rotation speeds but also heats up approximately 6 times more than the $\mathrm{Si}$ nanorods for the same input power. The low-aspect-ratio Au nanorod shows only slightly higher heating than the $\mathrm{Si}$, but it rotates about 4 times faster. To analyze this difference, we need to consider how particle size and shape influence the rotation frequency.

For a spheroidal particle, we have $f_{\text {rot }}=M_{\text {opt }} / 2 \pi \gamma_{\text {rot }}$, where $M_{\text {opt }} \propto P$ is the applied optical torque and $\gamma_{\text {rot }}=\pi g L^{3} \eta$ is the rotational friction coefficient. Here $g$ is a geometrical coefficient calculated from the particle length:width ratio $(g$ $=1$ for a sphere), $L$ is the length of the particle, and $\eta$ is the fluid viscosity as before. Table 1 shows that the $\mathrm{Si}$ nanorods and the low-aspect-ratio Au rods have similar $g$ values, which can therefore not explain the difference in rotation frequency. However, their lengths differ by $\sim 65 \%$ and this has a huge influence, since $f_{\text {rot }} \propto L^{-3}$. To reduce for the effects of dimensions, we now instead estimate how much optical torque the incident laser beam is able to transfer to the particle per unit power:

$$
\frac{\partial M_{\mathrm{opt}}}{\partial P}=\left.2 \pi^{2} g L^{3} \eta\left(T_{0}\right) \frac{\partial f_{\mathrm{rot}}}{\partial P}\right|_{P=0}
$$

This measure is relevant if the spinning particle is aimed to be used to drive a secondary particle or fluid to rotate, that is, if the particle is going to be used as a rotary nanomotor. ${ }^{34}$ As can be seen from Table 1 , the $\partial M_{\text {opt }} / \partial P$ differences between the particles are not as pronounced as the differences in absolute rotation frequency. In particular, it is clear that the Si nanorod performance is close to the low-aspect-ratio Au nanorod. This similarity can be qualitatively understood from the similarity in resonance anisotropy: The long-axis (short-axis) localized plasmon resonances of the $134 / 65 \mathrm{Au}$ nanorod occur at $\sim 700$ $\mathrm{nm}(550 \mathrm{~nm})$, resulting in a scattering anisotropy very similar to that produced through the anisotropic Mie resonances in the $\mathrm{Si}$ nanorod (Figure $1 \mathrm{~d}$ ) at the respective laser tweezer wavelengths. In contrast, the long-axis plasmon of the 173/65 
$\mathrm{Au}$ nanorod exactly overlaps with the trapping wavelength, thus amplifying the optical torque but also the amount of heat produced through resonant absorption. The results can also be compared to the maximum optical torque that can be extracted from a circularly polarized laser beam through scattering or absorption, $M_{\mathrm{opt}}^{\max }=\lambda_{\text {laser }} \mathrm{P} / 2 \pi c_{0}$, where $c_{0}$ is the speed of light. For the near-infrared laser wavelengths used to drive the $\mathrm{Si}$ and $\mathrm{Au}$ nanorods, this yields $\partial M_{\mathrm{opt}}^{\mathrm{max}} / \partial P \approx 4 \times 10^{-16} \mathrm{~N} \cdot \mathrm{m} / \mathrm{W}$. This is a factor $\sim 120$ higher than the actual torque extracted by the $\mathrm{Si}$ nanorod and $\sim 13$ times higher than that for the best $\mathrm{Au}$ nanorod, indicating that there is plenty of room for improving the efficiency of these nanoscale rotary motors.

\section{CONCLUSIONS}

We have shown that lithographically fabricated rod-shaped silicon nanoparticles can be released into aqueous solution and then optically trapped against a cover glass and rapidly rotated using circularly polarized laser tweezers. The maximum rotation frequency reached is $\sim 2 \mathrm{kHz}$, which is obtained for a relatively modest input power of $18 \mathrm{~mW}$ laser light with wavelength $\lambda_{\text {laser }}=785 \mathrm{~nm}$. Using two independent methods, Raman scattering and rotation analysis, we estimated the temperature increase of the trapped Si nanoparticles and their immediate surrounding to $\sim 3 \mathrm{~K} / \mathrm{mW}$ of incident laser power on average. Nanorods could be trapped and rotated with temperature excess down to $\sim 20 \mathrm{~K}$.

The rotational performance, quantified as the amount of optical torque that the Si particles extract from the incident beam, is approximately the same as previously reported for gold nanorods of similar aspect ratio and degree of resonance anisotropy. Nevertheless, it is likely that the Si performance could be substantially improved through further development of the fabrication methodology to yield more well-defined particle shapes and by optimizing the torque transfer efficiency by fine-tuning the Mie resonance properties of the particles. Moreover, photothermal heating could be further reduced by shifting the laser wavelength to $\lambda_{\text {laser }}=1064 \mathrm{~nm}$ where material absorption in $\mathrm{Si}$ is insignificant. This would, for example, make Si nanorods highly competitive as probes in microrheological measurements and as rotary nanomotors in future nanotechnology applications. The Raman thermometry technique is well-suited for combination with optical tweezers, but the signal level is low for wavelengths far from the direct band transitions in $\mathrm{Si}$, which occur in the near-UV. ${ }^{44}$ This could motivate the inclusion of a separate low-power shortwavelength Raman excitation source, though this would also complicate the experimental setup.

\section{METHODS}

Fabrication. Si nanorods were fabricated as described in refs 25 and 29. High-quality poly-silicon was first deposited on a fused silica wafer by low-pressure chemical vapor deposition (CVD), followed by deposition of a $100 \mathrm{~nm}$ tetraetyl orthosilicate (TEOS) film. The surface was then covered with $\mathrm{Ni}$ nanodisks (height $\times$ diameter $=40 \times 100 \mathrm{~nm}^{2}$ ) fabricated by hole-mask colloidal lithography. ${ }^{45}$ The TEOS layer not covered by $\mathrm{Ni}$ was then removed by reactive ion etching in a mixture of $\mathrm{CHF}_{3}$ and $\mathrm{Ar}$, after which the $\mathrm{Si}$ was subject to anisotropic etching by $\mathrm{Cl}$ gas at a pressure of 5 mTorr and an incident power of $50 \mathrm{~W}$. The Ni disk etch mask is then removed by wet etching in a Cr solution, after which the sample is rinsed in distilled water. The resulting Si particles can be released from the fused silica substrate by first dipping the sample in concentrated HF for $\sim 3 \mathrm{~min}$, followed by rinsing in abundant water, after which the etching is completed by drop casting $200 \mu \mathrm{L}$ of diluted HF $(0.1 \%)$ on the substrate until the removal of the particles can be confirmed by visible inspection of the substrate color. Possible HF residuals were removed by evaporation on a hot plate at $60^{\circ} \mathrm{C}$, after which the $\mathrm{Si}$ particles were redispersed in water, containing the surfactant cetyltrimethylammonium bromide (CTAB, $1 \mathrm{mM})$, to a particle concentration of the order $0.1 \mathrm{pM}$.

Optical Setup and Methodology. The optical tweezers setup was constructed around an inverted microscope (Nikon TE300) and a fiber-coupled diode laser (Toptica XTRA II 785 $\mathrm{HP}$ ) emitting at $785 \mathrm{~nm}$. The laser beam was made circularly polarized using a combination of wave-plates and then focused by the microscope objective (NA $=0.7$, Nikon CFI S Plan Fluor ELWD 60X) into the sample cell, consisting of two glass slides separated by a $100 \mu \mathrm{m}$ spacer. The sample was illuminated from above using an oil-immersion dark-field condenser (Nikon NA $=1.2-1.43$ ), which allowed for facile identification of individual nanoparticles in the sample cell. Backscattered laser light from a trapped nanoparticle was passed through a linear polarizer and recorded using a photomultiplier tube coupled to a hardware autocorrelator for photon correlation spectroscopy (PCS) rotational analysis. A second port of the microscope, equipped with a notch filter (Semrock $785 \mathrm{~nm}$ StopLine), allowed for collection of Raman spectra from trapped particles using a fiber coupled spectrometer (Shamrock 303i) equipped with a CMOS detector (Andor iDus). The same spectrometer could also be used for measuring elastic dark-field scattering spectra. The spectrometer system was carefully calibrated using dedicated halogen and mercury calibration lamps before each particle measurement. The particle suspension was diluted by a factor of $\sim 1000$ before experiments to avoid simultaneous trapping of several particles in the optical tweezers.

Electrodynamic Simulations. Optical cross sections (Figure 1) of the Si nanoparticles were obtained from finite difference time-domain (FDTD) simulations using commercially available software (Lumerical Solutions, Inc. Canada) using a total field scattered field excitation source. The particles were modeled as cylinders with variable diameter $D$ and height $h$ and with incident polarization either along or perpendicular to the rotational symmetry axis. The experimentally measured dielectric function of poly- $\mathrm{Si}^{46}$ was used in the simulations.

\section{ASSOCIATED CONTENT}

\section{Supporting Information}

The Supporting Information is available free of charge at https://pubs.acs.org/doi/10.1021/acs.nanolett.0c02240.

Experimental data of additional silicon nanoparticles and statistics, Brownian motion decay time plot, and calibration for the phonon Raman scattering thermometry (PDF)

\section{AUTHOR INFORMATION}

\section{Corresponding Authors}

Pawel Karpinski - Department of Physics, Chalmers University of Technology, Gothenburg, Sweden; Chemistry Department, Wroclaw University of Science and Technology, Wroclaw, Poland; orcid.org/0000-0001-8257-6940;

Email: pawel.karpinski@pwr.edu.pl 
Mikael Käll - Department of Physics, Chalmers University of Technology, Gothenburg, Sweden; () orcid.org/0000-00021163-0345; Email: mikael.kall@chalmers.se

\section{Authors}

Steven Jones - Department of Physics, Chalmers University of Technology, Gothenburg, Sweden; 이이이.org/0000-00032813-1945

Hana Sípová-Jungová - Department of Physics, Chalmers University of Technology, Gothenburg, Sweden; 이이.org/ 0000-0002-5383-9120

Ruggero Verre - Department of Physics, Chalmers University of Technology, Gothenburg, Sweden; 이이이.org/0000-00018337-9009

Complete contact information is available at:

https://pubs.acs.org/10.1021/acs.nanolett.0c02240

\section{Notes}

The authors declare no competing financial interest.

\section{ACKNOWLEDGMENTS}

This work was funded by the Knut and Alice Wallenberg Foundation, the Swedish Research Council, and the Chalmers Excellence Initiative Nano. P.K. would like to acknowledge National Science Centre, Poland, for financial support under POLONEZ Project No. 2016/23/P/ST3/02156, which has received funding from the European Union's Horizon 2020 research and innovation program under the Marie SkłodowskaCurie Grant Agreement No. 665778.

\section{REFERENCES}

(1) Svoboda, K.; Block, S. M. Optical Trapping of Metallic Rayleigh Particles. Opt. Lett. 1994, 19 (13), 930-932.

(2) Lehmuskero, A.; Johansson, P.; Rubinsztein-Dunlop, H.; Tong, L.; Käll, M. Laser Trapping of Colloidal Metal Nanoparticles. ACS Nano 2015, 9 (4), 3453-3469.

(3) Hirsch, L. R.; Stafford, R. J.; Bankson, J. A.; Sershen, S. R.; Rivera, B.; Price, R. E.; Hazle, J. D.; Halas, N. J.; West, J. L. NanoshellMediated near-Infrared Thermal Therapy of Tumors under Magnetic Resonance Guidance. Proc. Natl. Acad. Sci. U. S. A. 2003, 100 (23), $13549-13554$

(4) Huang, X.; El-Sayed, I. H.; Qian, W.; El-Sayed, M. A. Cancer Cell Imaging and Photothermal Therapy in the Near-Infrared Region by Using Gold Nanorods. J. Am. Chem. Soc. 2006, 128 (6), 21152120.

(5) Anker, J. N.; Hall, W. P.; Lyandres, O.; Shah, N. C.; Zhao, J.; Van Duyne, R. P. Biosensing with Plasmonic Nanosensors. Nat. Mater. 2008, 7 (6), 442-453.

(6) Paven, M.; Mayama, H.; Sekido, T.; Butt, H. J.; Nakamura, Y.; Fujii, S. Light-Driven Delivery and Release of Materials Using Liquid Marbles. Adv. Funct. Mater. 2016, 26 (19), 3199-3206.

(7) Xin, H.; Li, B. Targeted Delivery and Controllable Release of Nanoparticles Using a Defect-Decorated Optical Nanofiber. Opt. Express 2011, 19 (14), 13285-13290.

(8) Maier, C. M.; Huergo, M. A.; Milosevic, S.; Pernpeintner, C.; Li, M.; Singh, D. P.; Walker, D.; Fischer, P.; Feldmann, J.; Lohmüller, T. Optical and Thermophoretic Control of Janus Nanopen Injection into Living Cells. Nano Lett. 2018, 18 (12), 7935-7941.

(9) Hansen, P. M.; Bhatia, V. K.; Harrit, N.; Oddershede, L. Expanding the Optical Trapping Range of Gold Nanoparticles. Nano Lett. 2005, 5 (10), 1937-1942.

(10) Lehmuskero, A.; Ogier, R.; Gschneidtner, T.; Johansson, P.; Käll, M. Ultrafast Spinning of Gold Nanoparticles in Water Using Circularly Polarized Light. Nano Lett. 2013, 13, 3129-3134.
(11) Baffou, G.; Quidant, R.; García De Abajo, F. J. Nanoscale Control of Optical Heating in Complex Plasmonic Systems. ACS Nano 2010, 4 (2), 709-716.

(12) Baffou, G.; Quidant, R. Thermo-Plasmonics: Using Metallic Nanostructures as Nano-Sources of Heat. Laser and Photonics Reviews 2013, 7, 171-187.

(13) Fang, Z.; Zhen, Y. R.; Neumann, O.; Polman, A.; García De Abajo, F. J.; Nordlander, P.; Halas, N. J. Evolution of Light-Induced Vapor Generation at a Liquid-Immersed Metallic Nanoparticle. Nano Lett. 2013, 13 (4), 1736-1742.

(14) Hou, L.; Yorulmaz, M.; Verhart, N. R.; Orrit, M. Explosive Formation and Dynamics of Vapor Nanobubbles around a Continuously Heated Gold Nanosphere. New J. Phys. 2015, 17, 013050 .

(15) Andrén, D.; Shao, L.; Odebo Länk, N.; Aćimović, S. S.; Johansson, P.; Käll, M. Probing Photothermal Effects on Optically Trapped Gold Nanorods by Simultaneous Plasmon Spectroscopy and Brownian Dynamics Analysis. ACS Nano 2017, 11 (10), 1005310061.

(16) Jones, S.; Andrén, D.; Antosiewicz, T. J.; Käll, M. Ultrafast Modulation of Thermoplasmonic Nanobubbles in Water. Nano Lett. 2019, 19 (11), 8294-8302.

(17) Huang, X.; Jain, P. K.; El-Sayed, I. H.; El-Sayed, M. A. Plasmonic Photothermal Therapy (PPTT) Using Gold Nanoparticles. Lasers Med. Sci. 2008, 23, 217.

(18) Kuznetsov, A. I.; Miroshnichenko, A. E.; Brongersma, M. L.; Kivshar, Y. S.; Luk'yanchuk, B. Optically Resonant Dielectric Nanostructures. Science (Washington, DC, U. S.) 2016, 354 (6314), No. aag2472.

(19) Jahani, S.; Jacob, Z. All-Dielectric Metamaterials. Nat. Nanotechnol. 2016, 11, 23-36.

(20) Pauzauskie, P. J.; Radenovic, A.; Trepagnier, E.; Shroff, H.; Yang, P.; Liphardt, J. Optical Trapping and Integration of Semiconductor Nanowire Assemblies in Water. Nat. Mater. 2006, 5, 97101.

(21) Irrera, A.; Artoni, P.; Saija, R.; Gucciardi, P. G.; Iatì, M. A.; Borghese, F.; Denti, P.; Iacona, F.; Priolo, F.; Maragò, O. M. SizeScaling in Optical Trapping of Silicon Nanowires. Nano Lett. 2011, 11 (11), 4879-4884.

(22) Donato, M. G.; Brzobohatý, O.; Simpson, S. H.; Irrera, A.; Leonardi, A. A.; Lo Faro, M. J.; Svak, V.; Maragò, O. M.; Zemánek, P. Optical Trapping, Optical Binding, and Rotational Dynamics of Silicon Nanowires in Counter-Propagating Beams. Nano Lett. 2019, 19 (1), 342-352.

(23) Andres-Arroyo, A.; Gupta, B.; Wang, F.; Gooding, J. J.; Reece, P. J. Optical Manipulation and Spectroscopy of Silicon Nanoparticles Exhibiting Dielectric Resonances. Nano Lett. 2016, 16 (3), 19031910.

(24) Donato, M. G.; Monaca, M. A.; Faggio, G.; De Stefano, L. D.; Jones, P. H.; Gucciardi, P. G.; Maragò, O. M. Optical Trapping of Porous Silicon Nanoparticles. Nanotechnology 2011, 22 (50), 505704.

(25) Verre, R.; Shao, L.; Odebo Länk, N.; Karpinski, P.; Yankovich, A. B.; Antosiewicz, T. J.; Olsson, E.; Käll, M. Metasurfaces and Colloidal Suspensions Composed of 3D Chiral Si Nanoresonators. Adv. Mater. 2017, 29 (29), 1701352.

(26) Maragò, O. M.; Jones, P. H.; Gucciardi, P. G.; Volpe, G.; Ferrari, A. C. Optical Trapping and Manipulation of Nanostructures. Nat. Nanotechnol. 2013, 8 (11), 807-819.

(27) Kuhn, S.; Kosloff, A.; Stickler, B. A.; Patolsky, F.; Hornberger, K.; Arndt, M.; Millen, J. Full Rotational Control of Levitated Silicon Nanorods. Optica 2017, 4, 356-360.

(28) Amoruso, S.; Bruzzese, R.; Spinelli, N.; Velotta, R.; Vitiello, M.; Wang, X.; Ausanio, G.; Iannotti, V.; Lanotte, L. Generation of Silicon Nanoparticles via Femtosecond Laser Ablation in Vacuum. Appl. Phys. Lett. 2004, 84, 4502 .

(29) Verre, R.; Odebo Länk, N.; Andrén, D.; Sípová, H.; Käll, M. Large-Scale Fabrication of Shaped High Index Dielectric Nanoparticles on a Substrate and in Solution. Adv. Opt. Mater. 2018, 6 (7), 1701253. 
(30) Ashkin, A.; Dziedzic, J. M.; Bjorkholm, J. E.; Chu, S. Observation of a Single-Beam Gradient Force Optical Trap for Dielectric Particles. Opt. Lett. 1986, 11 (5), 288-290.

(31) Yao, A.; Tassieri, M.; Padgett, M.; Cooper, J. Microrheology with Optical Tweezers. Lab Chip 2009, 9, 2568-2575.

(32) Zhang, S.; Gibson, L. J.; Stilgoe, A. B.; Favre-Bulle, I. A.; Nieminen, T. A.; Rubinsztein-Dunlop, H. Ultrasensitive Rotating Photonic Probes for Complex Biological Systems. Optica 2017, 4 (9), $1103-1108$

(33) Shao, L.; Yang, Z. J.; Andrén, D.; Johansson, P.; Käll, M. Gold Nanorod Rotary Motors Driven by Resonant Light Scattering. ACS Nano 2015, 9 (12), 12542-12551.

(34) Śípová-Jungová, H.; Andrén, D.; Jones, S.; Käll, M. Nanoscale Inorganic Motors Driven by Light: Principles, Realizations, and Opportunities. Chem. Rev. 2020, 120 (1), 269-287.

(35) Andrén, D.; Karpinski, P.; Käll, M. Construction and Operation of a Light-Driven Gold Nanorod Rotary Motor System. J. Visualized Exp. 2018, 136 (136), No. e57947.

(36) Hajizadeh, F.; Shao, L.; Andrén, D.; Johansson, P.; RubinszteinDunlop, H.; Käll, M. Brownian Fluctuations of an Optically Rotated Nanorod. Optica 2017, 4 (7), 746.

(37) Fogel'son, R. L.; Likhachev, E. R. Temperature Dependence of Viscosity. Tech. Phys. 2001, 46 (8), 1056-1059.

(38) Balkanski, M.; Wallis, R. F.; Haro, E. Anharmonic Effects in Light Scattering Due to Optical Phonons in Silicon. Phys. Rev. B: Condens. Matter Mater. Phys. 1983, 28 (4), 1928-1934.

(39) Zograf, G. P.; Petrov, M. I.; Zuev, D. A.; Dmitriev, P. A.; Milichko, V. A.; Makarov, S. V.; Belov, P. A. Resonant Nonplasmonic Nanoparticles for Efficient Temperature-Feedback Optical Heating. Nano Lett. 2017, 17 (5), 2945-2952.

(40) Gogotsi, Y.; Baek, C.; Kirscht, F. Raman Microspectroscopy Study of Processing-Induced Phase Transformations and Residual Stress in Silicon. Semicond. Sci. Technol. 1999, 14 (10), 936-944.

(41) Lucazeau, G.; Abello, L. Micro-Raman Analysis of Residual Stresses and Phase Transformations in Crystalline Silicon under Microindentation. J. Mater. Res. 1997, 12 (9), 2262-2273.

(42) Rings, D.; Chakraborty, D.; Kroy, K. Rotational Hot Brownian Motion. New J. Phys. 2012, 14, 053012.

(43) Arita, Y.; Richards, J. M.; Mazilu, M.; Spalding, G. C.; Skelton Spesyvtseva, S. E.; Craig, D.; Dholakia, K. Rotational Dynamics and Heating of Trapped Nanovaterite Particles. ACS Nano 2016, 10, 11505-11510.

(44) Renucci, J. B.; Tyte, R. N.; Cardona, M. Resonant Raman Scattering in Silicon. Phys. Rev. B 1975, 11 (10), 3885-3895.

(45) Fredriksson, H.; Alaverdyan, Y.; Dmitriev, A.; Langhammer, C.; Sutherland, D. S.; Zäch, M.; Kasemo, B. Hole-Mask Colloidal Lithography. Adv. Mater. 2007, 19, 4297-4302.

(46) Maimaiti, A.; Patra, P. P.; Jones, S.; Antosiewicz, T. J.; Verre, R. Low-Loss Hybrid High-Index Dielectric Particles on a Mirror for Extreme Light Confinement. Adv. Opt. Mater. 2020, 8, 1901820. 